\title{
Oferta educativa para un bachillerato en mandarín ${ }^{1}$
}

\section{(Curriculum Proposal for a Bachelor's in Mandarin)}

\section{Vivian Vargas Barquero ${ }^{2}$}

Universidad Nacional, Costa Rica

\section{Olga Chaves Carballo}

Universidad Nacional, Costa Rica

\begin{abstract}
RESUMEN
En este artículo se analizan y comentan alternativas para el diseño de un plan de estudios para la enseñanza del mandarín, desde la academia costarricense. Por la creciente importancia de las relaciones bilaterales, de índole política, comercial y cultural con China, se señala la pertinencia de promover el diseño de un bachillerato en mandarín como parte de la oferta académica en la Universidad Nacional (Costa Rica). Para ello, se expone el proceso llevado a cabo para poner en marcha la propuesta, lo que incluye un pormenorizado informe de los resultados de encuestas entre el sector meta. De ello, además, se desprenden diversas recomendaciones, en materia pedagógica, logística e institucional en general.
\end{abstract}

\section{Abstract}

This article analyzes and discusses alternatives for the design of a curriculum for the teaching of Mandarin, from a Costa Rican academic perspective. Due to the increasing importance of bilateral political, commercial and

1 Recibido: 27 de mayo de 2017; aceptado: 2 de mayo de 2018. Una versión anterior de este artículo (Vivian Vargas Barquero y Olga Chaves Carballo, «La propuesta de un Bachillerato en Mandarín en la UNA») se difundió durante el $V$ Congreso Internacional de Lingüística Aplicada (V CI$L A P)$, del 5, 6 y 7 de 2016 (Universidad Nacional, Campus Omar Dengo, Heredia, Costa Rica), CD-ROM.

2 Escuela de Literatura y Ciencias del Lenguaje. Correo electrónico: vivian.vargas.barquero@una.cr

3 Escuela de Literatura y Ciencias del Lenguaje. Correo electrónico: profeolgachaves@hotmail.com

LETRAS 63 (2018), ISSN 1409-424X; EISSN 2215-4094

Doi: http://dx.doi.org/10.15359/rl.1-63.8

www.revistas.una.ac.cr/index.php/letras 
cultural relations with China, the relevance of promoting the design of a bachelor's degree in Mandarin, as one of the programs offered by the Universidad Nacional (Costa Rica), is highlighted. For this purpose, the process carried out to initiate the proposal is described, including a detailed report of the results of surveys among the target sector. In addition, various recommendations are given, in pedagogical, logistical and institutional matters in general.

Palabras clave: enseñanza de lenguas, enseñanza del mandarín, mandarín en Costa Rica

Keywords: language teaching, teaching of Mandarin, Mandarin in Costa Rica

\section{Introducción}

En 2011 se originó el proyecto de un bachillerato en mandarín, con la asesoría de la Embajada de China, debido al interés en hablar mandarín. Para dar respuesta al auge creciente en el mercado laboral, la Asamblea de académicos de la Escuela de Literatura y Ciencias del Lenguaje (ELCL) designó una comisión en 2012, para elaborar la propuesta de un bachillerato en mandarín. Esta comisión se propuso la tarea de investigar y crear un plan de estudios viable según las necesidades de la sociedad costarricense y latinoamericana, en general.

Por la importancia de las relaciones comerciales con la República Popular de China, el aprendizaje del mandarín es una prioridad en los centros educativos nacionales. Varias publicaciones recientes indican que «una de las razones principales que hacen útil el idioma mandarín es el ritmo al cual crecen las relaciones económicas de Costa Rica con China y con ello la necesidad que existe de que cada vez más costarricenses se relacionen con ciudadanos de ese país $\rangle^{4}$.

Como parte de la metodología, se realizó una lectura exhaustiva de publicaciones formales e informales acerca del tema y se plantearon las siguientes preguntas generadoras:

4 Noelia Solís, «Aprendizaje del mandarín llega a las escuelas costarricenses» (Xinhuanet, 2016) Web. 15 febrero 2016, <http://spanish.xinhuanet.com/2016-03/18/c_135200876.htm>. 
- ¿Cuáles son las necesidades y razones para ofrecer un bachillerato en mandarín en el país?

- ¿Cuál es la importancia y el auge de la enseñanza del mandarín en Costa Rica?

- ¿Cuál es el contexto socio-cultural y educativo en que se ofrecería el programa?

- ¿Cuáles son las propuestas de la UNA para responder a las necesidades de la sociedad?

- ¿Cuál es el interés que tienen los estudiantes por aprender mandarín?

- ¿Cuál es la metodología utilizada para la enseñanza de esta segunda lengua?

Para obtener esta información, se elaboró un cuestionario sobre el aprendizaje del mandarín, la motivación para estudiarlo, la metodología y las actividades utilizadas en la clase. Se aplicó a 38 estudiantes y cinco profesores de mandarín. Además, se efectuó una entrevista con la profesora Míngzi Lĭyáng, quien imparte cursos de mandarín en la ELCL.

Este estudio tiene el propósito de medir la percepción y pertinencia de la enseñanza del mandarín en la UNA. En segundo lugar, se propone una oferta académica para un bachillerato en mandarín. Incluye un análisis de la trayectoria histórica de la enseñanza del idioma en Costa Rica. Por el papel de esta carrera, esta investigación centra su análisis en aspectos como los procesos para el diseño del plan de estudios, desarrollo de la estructura curricular, los cursos y el trabajo de la comisión. En la última sección, se ofrecen recomendaciones de mediación pedagógica con el propósito de fortalecer la enseñanza del mandarín. 


\section{Contextualización}

Aspectos políticos, sociales, económicos y culturales en Costa Rica han llevado a los educadores a promover un ambiente con apropiadas opciones educativas para la enseñanza del mandarín. Sheng asevera que «hay un ambiente de gran recibimiento del idioma entre la población y tiene grandes prospectos de desarrollo» ${ }^{5}$, y también apunta que «el chino pasó de ser el idioma más difícil, el menos escogido entre los estudiantes, a ser considerado el idioma del futuro $»^{6}$. De la misma manera, hay informes que destacan las iniciativas y los programas que se han estado desarrollando en Costa Rica. Cada año es más notoria la apertura de programas de la enseñanza del mandarín, tanto en instituciones públicas como privadas. En los cuadros $1 \mathrm{a}$ y $1 \mathrm{~b}$ se muestran las acciones que se han gestado a favor de la enseñanza del mandarín en Costa Rica.

\section{Cuadro 1a. Reseña histórica de la enseñanza del mandarín en Costa Rica}

(2003-2012)

\begin{tabular}{|c|l|}
\hline Año & \multicolumn{1}{c|}{ Actividad } \\
\hline 2003 & La UNED inicia la enseña del mandarín en su Centro de Idiomas. \\
\hline 2005 & La UNA inicia la enseñanza del mandarín en la ELCL. \\
\hline 2006 & $\begin{array}{l}\text { El Colegio Victoria es el primer colegio privado en incorporar la } \\
\text { enseñanza del mandarín. }\end{array}$ \\
\hline 2009 & El Instituto Confucio (UCR) inicia sus labores. \\
\hline 2010 & $\begin{array}{l}\text { La Embajada de la R.P China abre su primer centro privado: El } \\
\text { Centro Cultural y Educativo Costarricense Chino. }\end{array}$ \\
\hline 2011 & La Falcon International School inicia clases en mandarín. \\
\hline 2012 & $\begin{array}{l}\text { La Asamblea de la ELCL emite un acuerdo para elaborar un plan de } \\
\text { estudios para un bachillerato en mandarín. }\end{array}$ \\
\hline
\end{tabular}

Fuente: elaboración propia, 2016.

5 José Sheng, Análisis del estado actual de la enseñanza y el modelo educativo del chino en Costa Rica (San José: Embajada de la República Popular China en Costa Rica, 2010) 12.

6 Sheng, 3 . 


\section{Cuadro 1b. Reseña Histórica de la Enseñanza del Mandarín en Costa Rica} (2013-2016)

\begin{tabular}{|c|l|}
\hline Año & \multicolumn{1}{|c|}{ Actividad } \\
\hline 2013 & $\begin{array}{l}\text { La Embajada de China realiza la primera convocatoria del } \\
\text { Programa de Cursos de Capacitación para el Profesorado de } \\
\text { Mandarín (MEP) en Costa Rica. } \\
\text { El Colegio Saint Gregory y el Colegio Nueva Esperanza inician la } \\
\text { enseñanza del mandarín. }\end{array}$ \\
\hline $2014-2015$ & El INA inicia un plan piloto en la enseñanza de mandarín. \\
\hline 2015 & $\begin{array}{l}\text { La Embajada de China apoya un nuevo proyecto de la enseñanza del } \\
\text { mandarín en centros de educación privada como el Colegio Victoria, } \\
\text { en Concepción de La Unión; el Colegio Internacional SEK, en } \\
\text { Cipreses de Curridabat; y el Colegio Saint Gregory de Tres Ríos. }\end{array}$ \\
\hline Marzo & $\begin{array}{l}\text { El Ministerio de Educación Pública (MEP) creó el Proyecto Red de } \\
\text { Colegios de Alta Oportunidad para ofrecer mandarín como tercer } \\
\text { idioma en algunos colegios: el Colegio de Bagaces, el Colegio } \\
\text { Técnico Profesional de Guácimo, Liceo Pacífico Sur, Colegio } \\
\text { Técnico Profesional Uladislao Gámez Solano, el Liceo Edgar } \\
\text { Cervantes y el Colegio Técnico Profesional de Purral. }\end{array}$ \\
\hline
\end{tabular}

Fuente: elaboración propia, 2016.

\section{La enseñanza del mandarín en la UNA}

Actualmente, la cooperación en los campos de la educación y la cultura requieren que la enseñanza del mandarín se desarrolle a un nivel superior. Debido a que la Embajada de la República Popular de China tiene interés en promover la enseñanza de este idioma y su cultura, colabora con profesores cooperantes del mandarín en la ELCL y se ha comprometido a seguir apoyando el Bachillerato en Mandarín en la UNA. La ELCL ha impartido cursos de mandarín desde 2005, que incluyen los integrales del nivel 1 al 6 y se ofrecen a la comunidad universitaria como cursos opcionales que tienen una alta demanda. En 2016, 107 estudiantes se inscribieron ${ }^{7}$. Por esta razón, la UNA dispuso

7 May Ling González, Datos suministrados por la Oficina de Coordinación de Cursos de Servicio (Heredia: ELCL, Universidad Nacional, 2016). 
una oferta educativa para la enseñanza del mandarín, con el apoyo logístico y coordinación académica, aprovechando el acompañamiento del Ministerio de Educación Pública (MEP) y Hanban (Oficina Nacional de Promoción Internacional de la Lengua China (国家汉办), organismo que se dedica a promover la enseñanza del idioma y su cultura.).

El contexto del aprendizaje de idiomas ha cambiado en el mundo contemporáneo; los alumnos de hoy día deben adquirir competencias lingüísticas nuevas y diversas que, en especial, faciliten la comunicación entre las comunidades y entre los países ${ }^{8}$. El objetivo es propiciar habilidades lingüísticas de la lengua meta para desempeñarse en campos del comercio internacional, economía, política y la enseñanza y traducción del mandarín. Teniendo como objetivo la creación de conocimiento, la ELCL incursiona en el desarrollo social, político y cultural del país para la enseñanza del mandarín con un plan de estudios avalado por expertos en la enseñanza de idiomas y con garantías de calidad para cumplir las metas propuestas por la educación costarricense ${ }^{9}$.

\section{El mandarín en Latinoamérica}

En la revisión bibliográfica pertinente para elaborar el plan de estudios, se concluyó que no se tiene noticia de algún plan de estudios a nivel de bachillerato en Latinoamérica. Aunque se cuenta con el apoyo de las embajadas de la República Popular de China, no existen programas de grado a nivel universitario. En la educación superior, cada vez se imparten más cursos libres de idiomas o como requisito para otras carreras. Esto es un indicador del auge que tiene este idioma en la educación latinoamericana que responde al comercio, industria y economía mundial. Uno de estos esfuerzos es la declaración de la

8 UNESCO e International Conference of Languages, Mejorar la competencia lingüistica y la enseñanza de idiomas. (Suzhou, China: Conferencia Internacional sobre las Lenguas, 2014: 3).

9 Olga Chaves, Jimmy Ramírez, Oscar Rojas, Ileana Saborío y Vivian Vargas, Plan de Estudios del Bachillerato en Mandarin (Heredia: Escuela de Literatura y Ciencias del Lenguaje, 2015) 8. 
embajada de Nicaragua ${ }^{10}$, el cual resalta que el desplazamiento del equilibrio de poder de la economía mundial ha despertado un enorme interés en conocer y aprender el chino o mandarín.

El chino mandarín está cobrando fuerza en el mundo. Los académicos y ejecutivos de la región tienen claro que aumentar las capacidades profesionales y humanas para enfrentarse al desafío que impone el comercio con el gigante asiático es, en la actualidad, algo fundamenta ${ }^{11}$. Las iniciativas para enseñar el mandarín en países latinoamericanos son variadas y siguen en aumento. A continuación se detallan las principales iniciativas de difusión de ese idioma. En primer lugar, la oficina general del Instituto Confucio (Hanban) cuenta con 29 centros educativos en América Latina. El primero inició en México en 2006 y el resto de estos institutos se encuentran en Ecuador, Perú, Chile, Argentina, Cuba, Colombia, México, Brasil, Bolivia y Costa Rica $^{12}$. Adicionalmente, en 2014 Chile promovió la enseñanza del mandarín, otorgando becas virtuales por medio del Instituto Nacional de Juventud. Por su parte, Colombia funda el Centro China para impartir cursos de mandarín. Uruguay y Costa Rica ya cuentan con el apoyo de sus gobiernos para impulsar clases de chino en escuelas públicas. Esta trayectoria resalta el impacto positivo hacia el aprendizaje del mandarín en estas regiones latinoamericanas.

\section{Sistematización de experiencias de la Comisión de diseño del plan de estudios}

La ELCL designó una comisión para el diseño de un bachillerato en mandarín (sesión ordinaria celebrada el 23 de mayo de

10 Embajada de Nicaragua, «La importancia mundial del idioma mandarín», Diario La Jornada Net (Nicaragua), 13 agosto 2015, <http://www.lajornadanet.com/diario/archivo/2010/enero/6/9.html >.

11 Cristina Vílchez, «Chino mandarín: el nuevo idioma de los negocios», MBA America Economía. americaeconomia. (MBA \& Educación Ejecutiva, 2015) Web. 12 agosto 2015, < http://mba.americaeconomia.com/articulos/reportajes/chino-mandarin-el-nuevo-idioma-de-los-negocios>.

12 Kongzi Xue Yuan, «Confucius Classroom 347» (Hanban.org. 9 de agosto de 2016) Web 17 de agosto de $2016<\mathrm{http}: / /$ english.hanban.org/node_10971.htm>. 
2012, acta n. ${ }^{\circ}$ 08-2012). Esta comisión inició la elaboración del plan de estudios investigando acerca de la importancia del mandarín en Latinoamérica. Seguidamente, se diseñó la estructura curricular con la cooperación de profesores expertos en la enseñanza del mandarín y con la asesoría externa de un especialista en el área. El desarrollo de destrezas para comunicarse efectivamente en este idioma meta se describe de la siguiente manera.

Este Bachillerato tiene como objetivo el desarrollo integral de las destrezas lingüísticas de la lengua meta en aquellos estudiantes que, por su perfil profesional, requieran del dominio del idioma para desempeñarse laboralmente en campos del comercio internacional, economía, política, enseñanza y traducción del mandarín ${ }^{13}$.

En cuadros $2 \mathrm{a}, 2 \mathrm{~b}$ y $2 \mathrm{c}$, se han resumido las principales acciones realizadas para la viabilidad de la nueva oferta académica, a cargo de la comisión del diseño del Bachillerato en Mandarín.

13 Chaves y otros, 7 . 
Cuadro 2a. Sistematización de acciones realizadas para la viabilidad de la nueva oferta académica (2013-2015)

\begin{tabular}{|c|l|l|}
\hline Fecha & \multicolumn{1}{|c|}{ Actividad } & \multicolumn{1}{c|}{ Propósito } \\
\hline 2013 & $\begin{array}{l}\text { Reunión con Yang Ming } \\
\text { (Agregado de Educación de } \\
\text { la Embajada China en Costa } \\
\text { Rica) }\end{array}$ & $\begin{array}{l}\text { Continuar con el convenio que } \\
\text { se había establecido desde que se } \\
\text { iniciaron los cursos de servicio en } \\
\text { mandarín en 2005 para una nueva } \\
\text { oferta de un bachillerato en mandarín. } \\
\text { Negociar el apoyo que nos brindará la } \\
\text { Embajada en este diseño. }\end{array}$ \\
\hline 2012 & $\begin{array}{l}\text { Solicitud de estudio de } \\
\text { viabilidad realizado por } \\
\text { el Instituto de Estudios } \\
\text { Sociales en Población } \\
\text { (IDESPO) }\end{array}$ & $\begin{array}{l}\text { Conocer el tipo de población } \\
\text { interesada en cursar el bachillerato y } \\
\text { el posible número de estudiantes de } \\
\text { primer ingreso a esta carrera. }\end{array}$ \\
\hline $2014-2015$ & $\begin{array}{l}\text { Reunión con el Decano de } \\
\text { la Facultad de Filosofía y } \\
\text { Letras, Albino Chacón }\end{array}$ & $\begin{array}{l}\text { Reforzar el compromiso de Facultad } \\
\text { para la apertura de esta nueva oferta } \\
\text { académica. }\end{array}$ \\
\hline
\end{tabular}

Fuente: elaboración propia, 2016.

Cuadro 2b. Sistematización de acciones realizadas para la viabilidad de la nueva oferta académica (agosto 2014-marzo 2015)

\begin{tabular}{|c|l|l|}
\hline Fecha & \multicolumn{1}{|c|}{ Actividad } & \multicolumn{1}{c|}{ Propósito } \\
\hline $\begin{array}{c}\text { agosto de } \\
2014\end{array}$ & $\begin{array}{l}\text { Reunión con Sandra León } \\
\text { (Rectora de UNA) }\end{array}$ & $\begin{array}{l}\text { Socializar el avance de la elaboración } \\
\text { del plan de estudios y la sostenibilidad } \\
\text { en la que se compromete la Rectoría. }\end{array}$ \\
\hline & & $\begin{array}{l}\text { Posibilidades de cooperación: } \\
\text { a. Apoyo de BISU para el desarrollo } \\
\text { del Bachillerato en Mandarín. }\end{array}$ \\
$\begin{array}{c}\text { marzo de de } \\
2015\end{array}$ & $\begin{array}{l}\text { Visita de la delegación de } \\
\text { la Universidad de Estudios } \\
\text { Internacionales, BISU }\end{array}$ & $\begin{array}{l}\text { b. Intercambio de profesores. } \\
\text { c. Materiales de apoyo a la docencia. } \\
\text { d. Intercambio de estudiantes. } \\
\text { e. } \begin{array}{l}\text { Negociaciones para obtención } \\
\text { de doble titulación para los } \\
\text { estudiantes. }\end{array}\end{array}$ \\
\hline
\end{tabular}

Fuente: elaboración propia, 2016. 
Tabla 2c. Sistematización de acciones realizadas para la viabilidad de la nueva oferta académica (noviembre 2014-julio 2016)

\begin{tabular}{|c|c|c|}
\hline Fecha & Actividad & Propósito \\
\hline $\begin{array}{l}\text { nov. de } \\
2014\end{array}$ & $\begin{array}{l}\text { Esfuerzo para la elaboración } \\
\text { de los términos de un } \\
\text { convenio entre la Universidad } \\
\text { de Hanban y la UNA. Con } \\
\text { la colaboración de la Oficina } \\
\text { de Cooperación Técnica } \\
\text { Internacional se redacta dicho } \\
\text { convenio. }\end{array}$ & $\begin{array}{l}\text { Buscar cooperación entre ambas } \\
\text { universidades como apoyo sostenible } \\
\text { al Bachillerato en Mandarín en } \\
\text { términos de presupuesto para compra } \\
\text { de laboratorio de idiomas, materiales, } \\
\text { becas para estudiantes y profesores } \\
\text { provenientes de China. }\end{array}$ \\
\hline 2015 & $\begin{array}{l}\text { Acuerdo de la Asamblea de la } \\
\text { ELCL }\end{array}$ & $\begin{array}{l}\text { Aprobación de los cursos del V y VI } \\
\text { nivel del mandarín. }\end{array}$ \\
\hline $\begin{array}{l}8 \text { de feb. } \\
\text { de } 2016\end{array}$ & $\begin{array}{l}\text { Envío de borrador final del } \\
\text { plan de estudios al Proceso } \\
\text { de Diseño e Innovación } \\
\text { Curricular }\end{array}$ & $\begin{array}{l}\text { Después de un arduo trabajo de } 4 \\
\text { años de diseño y revisión del plan de } \\
\text { estudio, se hace entrega. }\end{array}$ \\
\hline $\begin{array}{l}\text { abril de } \\
2016\end{array}$ & $\begin{array}{l}\text { Acuerdo de Vicerrectoría de } \\
\text { Docencia }\end{array}$ & $\begin{array}{l}\text { Se indica que el plan de estudio } \\
\text { ya puede ser enviado a CONARE } \\
\text { después de que se asigne jornadas } \\
\text { académicas y se apruebe en la } \\
\text { Asamblea de académicos de ELCL. }\end{array}$ \\
\hline $\begin{array}{l}\text { julio de } \\
2016\end{array}$ & $\begin{array}{l}\text { Visita con Wan Zhijia } \\
\text { (Agregado de Educación de } \\
\text { la Embajada China en Costa } \\
\text { Rica) }\end{array}$ & $\begin{array}{l}\text { Se retoma con la Rectoría y el } \\
\text { decanato las conversaciones acerca } \\
\text { del apoyo presupuestario. Se les } \\
\text { presenta la malla curricular y el plan } \\
\text { de estudios finalizado. }\end{array}$ \\
\hline
\end{tabular}

Fuente: elaboración propia, 2016.

Aprovechando la experiencia de los académicos de la comisión en la enseñanza de un segundo idioma, se preparó el plan de estudios el cual tiene una viabilidad favorable con el compromiso de las autoridades universitarias, la Embajada de China y la cooperación de universidades de la República Popular de China. Incluye cuatro ejes curriculares: a) conocimientos lingüísticos, b) desarrollo de las habilidades lingüísticas, c) comunicación intercultural y d) competencia cultural. 
Para completar el plan, se efectuaron sesiones semanales de trabajo; además, se realizó trabajo individual para el diseño de cursos, revisión y edición de los diferentes componentes del plan de estudios, junto con otras reuniones periódicas con autoridades en la enseñanza del mandarín y cooperantes internacionales. Dos integrantes de esta comisión han realizado cursos de mandarín básico con el propósito de observar el aprendizaje de este idioma en la clase, analizar la metodología empleada por los profesores y, en general, para conocimiento profesional. Este aprendizaje se utilizó como insumo en la elaboración de la estructura curricular. Con base en ello y como integrantes de la comisión, las investigadoras realizaron un estudio de percepciones de los estudiantes y los profesores de la UNA, acerca del aprendizaje y la pertinencia del mandarín, lo que refleja cómo la nueva oferta es una necesidad latinoamericana.

\section{Proceso metodológico (tipo de investigación, población/sujetos, descripción de instrumento)}

Esta investigación es un estudio de percepción de estudiantes y profesores de los cursos de idiomas para otras carreras, acerca del aprendizaje y la pertinencia de la enseñanza del mandarín en la UNA. Se realizó un estudio en el primer ciclo lectivo de 2016. El sector participante incluye cinco profesores de China y 38 estudiantes de los niveles 1,2 y 3 . La edad de la población estudiantil encuestada se encuentra en un rango de 17 a 25 años. Todos estaban matriculados en distintas carreras de la UNA y su motivación era principalmente cumplir un requisito de créditos en un idioma extranjero, para aprender otro idioma diferente al inglés, porque encuentran dificultad en ese idioma o por una motivación o afinidad genuina hacia el aprendizaje del mandarín. La edad de los profesores es de entre 25 a 30 años.

La escogencia de la población estudiantil se hizo conforme a cinco aspectos: primeramente, es una población universitaria con características similares a los futuros estudiantes del bachillerato 
propuesto; por lo tanto, en el currículo universitario, se puede medir las destrezas, la metodología, las actividades de clase y la motivación que son los factores influyentes en el aprendizaje de esta lengua. En segundo lugar, en el contexto costarricense la enseñanza el mandarín se efectúa a nivel de primaria, secundaria y en otros centros de idiomas como el instituto Confucio y el Centro Cultural, por lo que no es práctico realizar encuestas con esta población, ya que su realidad educativa es muy diferente a la que se da en la enseñanza superior. En tercer lugar, los estudiantes universitarios que ya tienen experiencia en el aprendizaje de este idioma pueden dar fe del grado de dificultad o facilidad para aprender el mandarín. En cuarto lugar, no hay otra población a la que se pueda acceder con encuestas, ya que las personas que hablan mandarín son hablantes nativos o lo aprendieron en el extranjero. Por último, las investigadoras son expertas en la enseñanza $\mathrm{y}$ aprendizaje de segundas lenguas, lo que las habilita para realizar este tipo de pesquisa con la población seleccionada.

Se diseñó un instrumento para estudiantes y docentes. El de estudiantes consta de dos partes: cinco preguntas cerradas, las cuales se construyeron de acuerdo con la escala de Likert; estas contemplan los siguientes rubros: excelente, bueno, promedio, deficiente y debe mejorar; también, incluye cinco preguntas abiertas que miden sus impresiones, las cuales se analizan anónimamente. Se aplicó la encuesta a un total de 38 estudiantes del primero, segundo y tercer nivel. El procedimiento en la aplicación del instrumento consistió en una versión impresa la cual se entregó a los estudiantes durante el curso y se les dio aproximadamente treinta minutos para completarlo.

El instrumento se adaptó para efectuar encuestas por vía electrónica a seis profesores que han impartido cursos de mandarín en la UNA. Cuatro de ellos provienen de la República Popular de China y uno es costarricense; todos cuentan con un bachillerato en la enseñanza del mandarín de la República Popular de China. Este instrumento está dividido en dos partes. La primera corresponde a siete preguntas con la escala Likert que contemplan los siguientes rubros: mucho, 
suficiente, algo, poco y muy poco. La segunda parte consta de cinco preguntas abiertas y una pregunta de selección. Además, se realizó una entrevista a una profesora de mandarín para indagar sobre la metodología utilizada para la enseñanza de este idioma.

\section{Resultados y análisis}

En este primer apartado se analizan y se ilustran los resultados de las impresiones de 38 estudiantes. En el primer nivel se efectuó la encuesta a 16 estudiantes, en el segundo a 17 estudiantes y a cinco en el tercer nivel. La primera parte del cuestionario contempla los enunciados acerca de la enseñanza del idioma mandarín, el planeamiento de los cursos, las dinámicas desarrolladas en la clase y los beneficios e importancia del aprendizaje de este idioma. En el gráfico 1 se muestra el análisis de datos. En la primera pregunta 1, los estudiantes califican la enseñanza del idioma de una manera muy positiva destacando la excelencia de los cursos de mandarín.

\section{Gráfico 1. Valoración de excelencia del idioma mandarín en la UNA}

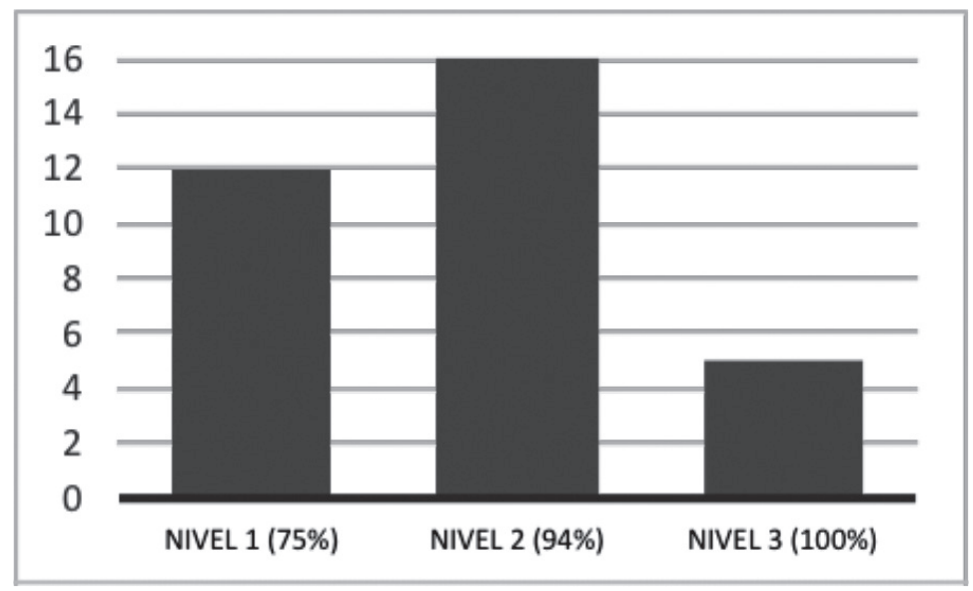

Fuente: Cuestionario (Anexo 1) 
En el gráfico 1, doce estudiantes de primer nivel, dieciséis estudiantes del segundo y todos del tercer nivel opinan que la enseñanza del mandarín está entre el rango de bueno y excelente. Las preguntas 2 y 3 miden el rango de excelencia del planeamiento y las dinámicas de clase. Los estudiantes opinan que en estos cursos aprenden sobre la cultura y la lengua. Además, en su opinión los cursos son intensivos, complejos y bien planeados.

\section{Gráfico 2. Planeamiento y dinámicas de los cursos de mandarín}

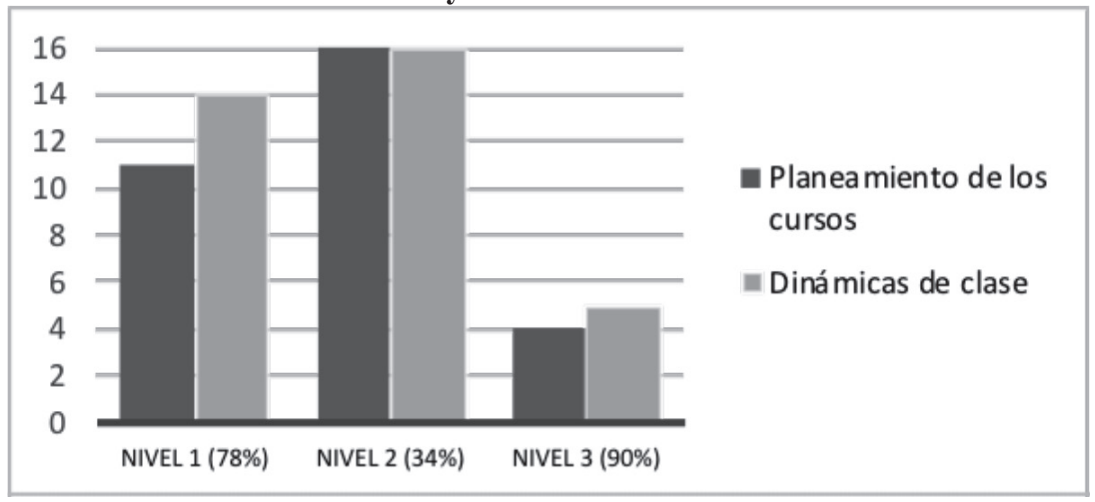

Fuente: Fuente: Cuestionario (Anexo 1)

El gráfico 2 muestra la excelencia de estos dos enunciados. En el primer nivel un 78\%, correspondiente a 11 y 14 estudiantes, estima que el planeamiento y las dinámicas de clase son excelentes. En el segundo nivel, un 34\% de los encuestados (16 personas) considera que el planeamiento y las dinámicas de clase son excelentes. El tercer nivel, el $90 \%$ (equivalente a 4 y 3 estudiantes) lo categoriza como excelente. Sin embargo, algunos de los comentarios de los estudiantes indican la necesidad de contar con más tiempo, más práctica de escritura de los caracteres, más material audiovisual, más dinámicas de clase y por último menor número de estudiantes en los cursos de primer y segundo nivel. 
Sobre las preguntas 4 y 5 , los estudiantes opinan que el idioma es indispensable para su formación profesional en la búsqueda de un trabajo, junto con el conocimiento de la cultura china y la comunicación intercultural. En general, los estudiantes concuerdan con la importancia que tiene el mandarín en el mercado laboral debido a la demanda existente de profesionales que dominen este idioma. Este dominio no se alcanzaría llevando cursos de mandarín conversacional en las diferentes academias para el aprendizaje del idioma que existen en Costa Rica. Es esencial que los profesionales desarrollen un nivel de lengua superior para enfrentar las demandas que exige la economía mundial. Al respecto Sheng (2010) indica, los estudiantes «esperan que al estudiar chino puedan acceder en el futuro a mejores opciones de vida y de trabajo» ${ }^{14}$.

En la segunda parte del cuestionario, se analizan las estrategias de mediación pedagógica utilizadas por el profesor y los estudiantes, la motivación y las ventajas de aprender el idioma mandarín. En la pregunta 1 , los estudiantes manifiestan que entre las estrategias de mediación utilizadas están, en primer lugar, herramientas tecnológicas tales como celulares inteligentes, videos, y páginas web; en segundo lugar, la repetición y la memorización de las palabras y sonidos; en tercer lugar, la interacción con hablantes nativos del idiomay, en cuarto lugar, la práctica de escritura de caracteres y además, el aprendizaje sobre la cultura china. La pregunta 2, sobre la motivación para aprender el mandarín, se opina que es para conocer y ampliar el conocimiento de la cultura china, a desarrollar la afinidad por el idioma, a solicitar una beca en China o a viajar y prepararse para futuras relaciones laborales y superación personal.

En la pregunta 3, dentro de las actividades de clase más utilizadas para la promoción del aprendizaje del idioma, los estudiantes indican las siguientes actividades en orden de prioridad:

14 Sheng, 16. 
a. Dinámicas lúdicas para practicar la pronunciación

b. Práctica de palabras en la pizarra tanto en pinyin como en caracteres

c. Comprensión auditiva

d. Dinámicas grupales

e. Presentaciones orales y diálogos

f. Redacción de oraciones

g. Repetición de vocabulario

h. Traducción de vocabulario

i. Tareas extra clase

De la pregunta 4 , los estudiantes comentan que algunas de las actividades que menos contribuyen al aprendizaje son la escritura en la pizarra y prácticas con los compañeros sin instrucciones claras; adicionalmente, otras actividades que los estudiantes consideran más complejas incluyen: dictados, pruebas escritas, tareas sin guía y películas. Señalan que una de las debilidades del curso es la falta de variedad de actividades.

Finalmente, para la pregunta 5, entre las ventajas de aprender el mandarín los estudiantes incluyeron las opciones indicadas en el gráfico 3 .

Gráfico 3. Ventajas al aprender el mandarín

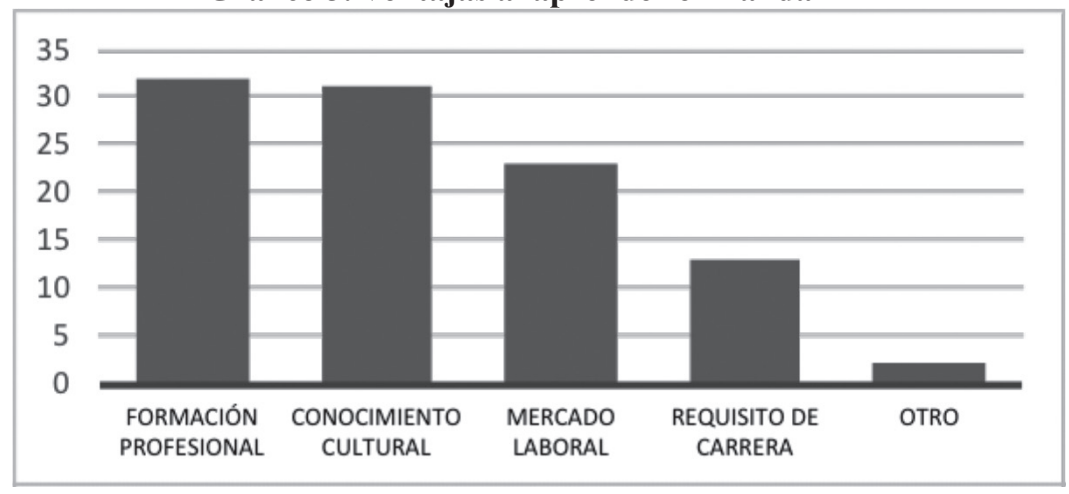

Fuente: Cuestionario (Anexo 1) 
El gráfico 3 clasifica las opiniones de los estudiantes sobre las ventajas del aprendizaje del mandarín. El gráfico muestra que 32 estudiantes consideran ventajoso el mandarín para su formación profesional, 31 de los estudiantes estiman el conocimiento cultural que obtienen una gran ventaja. Adicionalmente, 23 estudiantes encuentran el estudio del mandarín beneficioso para completar los requisitos de su carrera.

Estos resultados dan cuenta de la necesidad de la sociedad global, en que los aprendizajes deben ser multiculturales e interculturales; por tanto, un bachillerato en mandarín respondería a este nuevo cambio de paradigma en la educación. Para ilustrar, se ha seleccionado uno de los comentarios que los estudiantes de tercer nivel aportan apoyando la enseñanza del mandarín: «la UNA y Costa Rica deben invertir en enseñar mandarín como carrera para poder enseñarlo como segunda lengua en los colegios y escuelas».

El segundo instrumento de recolección de datos se aplicó a cinco profesores con experiencia en la enseñanza del mandarín en el programa de Cursos de Servicio de la UNA o el Instituto Confucio, siendo cuatro de ellos de China y el otro de Costa Rica. En la pregunta 1, estos encuestados opinan que están de acuerdo con la importancia de la enseñanza de este segundo idioma, puesto que se requiere comprender la cultura y el idioma para tratar con empresas chinas. Con respecto a las preguntas 2 y 3 , sobre la importancia que tiene la enseñanza del mandarín en el ámbito de la educación bilingüe costarricense, los cinco profesores concuerdan. Esto se ejemplifica en el siguiente comentario: «Cuando se aprende una nueva lengua, se adquiere también una nueva forma de pensar; la cultura china tiene una historia de más de cinco mil años. Incluir la enseñanza del mandarín en la educación costarricense sería un gran aporte para el desarrollo de los estudiantes que podrían enriquecerse con valores y enseñanzas de una cultura tan rica» (profesor costarricense con formación en China).

Para las preguntas 4 y 5 , se manifiesta que los beneficios del aprendizaje del mandarín se plasman en becas, mejores oportunidades 
de trabajo y oportunidades de viajar al extranjero ${ }^{15}$. En la pregunta 6 , cuatro de los profesores consideran que los centros que imparten mandarín cuentan con personal capacitado.

En la segunda parte se indagó sobre cómo logran motivar a los estudiantes, las actividades y las estrategias de mediación pedagógica utilizadas. En respuesta a la pregunta 1, los cinco profesores utilizan canciones, juegos, videos, transcripción de caracteres en papel original y con pincel, memorización de sonidos y caracteres, lecturas y diálogos cortos, dictados, ejercicios de comprensión auditiva, y tarjetas ilustrativas con vocabulario, herramientas tecnológicas, experiencias de inmersión como ir a un restaurante y ordenar en la lengua meta o simular interacciones similares en la clase y el uso del español en los primeros niveles, para motivar a los estudiantes.

Otras formas de motivación utilizadas incluyen la de conocer experiencias provechosas de otros estudiantes becados para continuar sus estudios del mandarín en China a través de becas y concursos como El puente chino ${ }^{16}$. Adicionalmente, las estrategias de aprendizaje que los profesores consideran menos útiles son: repeticiones descontextualizadas, corregir errores sin un objetivo claro, traducir palabras o frases cuando no es necesario, utilizar estructuras gramaticales complejas y evitar el uso de frases académicas en vez de frases coloquiales.

En cuanto al diseño de los cursos (preguntas 2 y 3 ), los profesores opinan que los cursos deben ser más sistemáticos, con objetivos alcanzables, específicos y apropiados al contexto costarricense. También, los profesores consideran que se debe hacer una mejor selección de materiales y búsqueda de recursos en línea, para solventar la carencia de material impreso. Dos profesores señalan la importancia de contar con más profesores capacitados para impartir este idioma y, aunque

15 Este instrumento es una adaptación del instrumento para los estudiantes; por lo tanto, ver instrumento que se aplicó a estudiantes.

16 Este es un concurso organizado por la Embajada de la República Popular de China, las universidades de imparten mandarín en Cursos de Servicio y el Instituto Confucio en Costa Rica. El concurso incluye presentaciones orales en mandarín y una presentación de un talento o destreza por parte de los concursantes. Los ganadores son becados con un viaje a China durante un mes. 
algunos son graduados, no tienen la suficiente experiencia en docencia en otros países como el nuestro. Finalmente, con respecto a las preguntas 4 y 5 , los profesores encuestados muestran conocimiento en la enseñanza del mandarín como segunda lengua y enseñan el mandarín de una forma atractiva para sensibilizar al estudiante en la comprensión de la cultura china y, por ende, el aprendizaje de este idioma.

En setiembre de 2016 se efectuó una entrevista con la profesora Míngzi Liyáng, acerca de la comparación y diferencia de la metodología de enseñanza aprendizaje utilizada en China y en Costa Rica. Expresa que la metodología empleada en Costa Rica es muy diferente a la que utiliza en China, porque la dinámica es más participativa en el contexto costarricense; los estudiantes utilizan más estrategias de aprendizaje dependiendo de su interés, estilo y nivel de lengua. Además, se espera que en una clase a nivel universitario existe más apertura a la discusión de temas sociales, políticos y culturales. Por esta razón, esta profesora expresó su disposición de recibir capacitación sobre estos aspectos didácticos.

Debido a la distancia, la publicidad, el poco acceso a la cultura, los medios de comunicación y las escasas oportunidades de interactuar con hablantes nativos, la enseñanza del mandarín como lengua extranjera es diferente de la de otros idiomas con los cuales los costarricenses están más familiarizados, Cook señala que los idiomas modernos presentan diversos grados de distancia interlingüística dependiendo de factores tales como parentesco, sustancia cultural y formas lingüísticas ${ }^{17}$. Esta secuencia de distancia interlingüística clasifica los idiomas según el grado de mutua inteligibilidad que estos posean, ubicando al mandarín y al español en dos extremos distantes. Se concluye entonces que la dificultad de un costarricense para aprender una lengua de la misma familia lingüística indoeuropea, como lo es el francés, es menor mientras que la dificultad es mayor al aprender un idioma de origen chino-tibetano como lo es el mandarín.

17 Guy Cook, Applied Linguistics (Nueva York: Oxford, 2010). 
Con base en lo anterior se concluye que dada la diferencia lingüística entre el español y el mandarín, este idioma requiere más practica, estudio y dedicación. A pesar de este hecho, las observaciones y experiencias de clase y los insumos de los estudiantes demuestran que, con esfuerzo, interés y entrega el estudiante puede cumplir con los objetivos del curso. La práctica constante de los tonos y de los caracteres, los materiales disponibles para cada nivel (audios, vídeos, películas, videojuegos), así como conversaciones con hablantes cuya lengua materna es el mandarín permiten al estudiante familiarizarse más con el idioma. Sin embargo, la metodología y las actividades de clase podrían ser aun más significativas y comunicativas, si se adoptaran otras estrategias que han sido útiles la enseñanza de otras lenguas extranjeras.

\section{Recomendaciones para el aprendizaje del mandarín}

A partir de los nuevos enfoques en metodología para la enseñanza de segundas lenguas, experiencias en la enseñanza-aprendizaje de una lengua extranjera y la observación en el aula como se ilustra en el cuadro 4, proponemos para el campo de la enseñanza del mandarín los siguientes comentarios y observaciones, con el fin de desarrollar un ambiente de aprendizaje cooperativo y exitoso.

A. Sobre la metodología empleada en los cursos de mandarín de la UNA, se observó que es estructural, memorística, y de repetición, por lo que se recomienda, capacitar a los docentes en formación pedagógica para que utilicen metodologías comunicativas, las TIC y adaptación de textos orales y escritos auténticos. Esta capacitación se puede hacer mediante la discusión de modelos de clase que reflejen prácticas comunicativas, cooperativas, dinámicas y creativas para la enseñanza de un idioma extranjero. 
B. Se espera que el profesor de mandarín diseñe un modelo de clase breve que incluya materiales, dinámicas y ejercicios. Para el diseño, adaptación y producción de materiales, se puede utilizar un texto ya publicado, material en línea o impreso como guía o referencia de trabajo en la creación de sus propias temáticas y ejercicios de caracteres chinos y en pinyin apropiados al nivel de lengua del estudiante y contextualizados a la realidad y los intereses culturales y sociales costarricenses.

C. Se recomienda un taller de inducción, con información pertinente al contexto de educación costarricense y las políticas administrativas y pedagógicas de la ELCL, que tenga en cuenta la población de estudiantes, sus características y sus necesidades, metodologías de evaluación, horarios de clase, uso de tecnologías, entre otros aspectos pedagógicos.

D. Otras recomendaciones como parte de la enseñanza integral y de la mediación pedagógica para fortalecer el aprendizaje y la enseñanza de esta lengua en la UNA:

1. Explorar la cultura china a través de visitas o actividades en clase con hablantes nativos y con otros estudiantes que han tenido una experiencia de inmersión en China.

2. Propiciar el uso de la memoria como estrategia de aprendizaje para recordar y reproducir frases cotidianas en el intercambio de información personal y básica, siempre y cuando se contextualice el idioma.

3. Utilizar diálogos cortos y situaciones de la vida cotidiana para presentaciones de temas personales: información de su vida, familia, gustos, educación, entretenimiento, tiempo libre y otros temas sociales y culturales.

4. Practicar la gramática con ejemplos contextualizados y aplicados a las necesidades inmediatas del estudiante.

5. Desarrollar material didáctico con ejercicios de comprensión auditiva, auténtica, corta y comprensible. 
6. Repasar el vocabulario en contextos reales a través de dinámicas en parejas o en grupos.

7. Incorporar una variedad de ejercicios para desarrollar las macro y micro destrezas del idioma.

Los insumos de esta investigación sirven de base para realizar una reflexión pedagógica e identificar el modelo de enseñanza más adecuado para la enseñanza del mandarín a nivel universitario. Para ello, se propone el diseño y la puesta en práctica de un taller de capacitación pedagógica y metodológica con el fin de garantizar la efectividad del aprendizaje de este idioma en el contexto costarricense. Este taller de capacitación pretende ir más allá del plan de estudios propuesto, para abarcar la mediación pedagógica, la metodología, las estrategias de enseñanza-aprendizaje, la evaluación, la evaluación, la selección y la elaboración de materiales.

\section{Conclusiones}

Queda demostrada la importancia de la enseñanza del mandarín en Costa Rica, tal como lo expresa Prado: «Es de esperar que, en un futuro no muy lejano, la región centroamericana se abra a las negociaciones con la República Popular China. Costa Rica está llamada a jugar un rol importante en abrir la brecha para facilitar y ejemplarizar el camino» ${ }^{18}$. La ELCL cuenta con una trayectoria en la enseñanza de este idioma; además, la cooperación con la Embajada de la República Popular de China ha contribuido exitosamente con esta meta. Por otra parte, la comisión de académicos especialistas de la ELCL elaboró el plan de estudios investigando, revisando la estructura curricular con profesores cooperantes de la Embajada de China.

18 Mimi Prado, «Presentación», Marta Trejos Montero, ed. Relaciones China Costa Rica. Una referencia para Centroamérica (San José: Asociación Instituto de Estudios Superiores para el Desarrollo Humano Sostenible, 2009) 6. Recuperado el 14 de marzo de 2016, <http://www19.iadb.org/ intal/intalcdi/PE/2011/07595.pdf>. 
La investigación empírica demuestra que la mayoría de los aprendientes resaltan la importancia de dedicar tiempo para estudiar este demandante idioma. Sin embargo, se puede evidenciar que el aprendizaje del mandarín es factible y amerita la consideración de una serie de aspectos curriculares como son: la mediación pedagógica, la metodología, el rol del profesor y el aprendiente, en el uso de los materiales, en la práctica, en el uso de las TIC y otros aspectos importantes del proceso enseñanza-aprendizaje.

Debido a la diferencia lingüística entre el mandarín y el español, se requiere más práctica, estudio y dedicación. Las observaciones y experiencias de clase y los comentarios de los estudiantes indican que con esfuerzo e interés se puede cumplir con los objetivos del curso. La práctica constante de los tonos y de los caracteres, los materiales disponibles para cada nivel (audios, videos, películas, videojuegos) y experiencias reales con hablantes nativos en supermercados y restaurantes permiten al estudiante acercarse y familiarizarse con esta lengua. Qiu Gui Su señala que con la práctica y el uso de la lengua se garantiza el éxito para utilizar el idioma en situaciones reales ${ }^{19}$.

Los estudiantes tienen el interés, muestran una actitud muy favorable para el aprendizaje, tienen la habilidad, y sienten que estos cursos han llenado sus expectativas en cuanto al idioma y la motivación como parte de su formación profesional. El estudio refleja claramente que la trayectoria de los cursos de mandarín; aunque haya limitaciones de tiempo, recursos y prácticas comunicativas, permiten el desarrollo lingüístico a un nivel básico y sobre todo motivan a los estudiantes a aprender de la cultura para continuar explorando otras experiencias culturales y académicas.

Los profesores también expresan necesidades para mejorar el diseño de los cursos y así continuar con la formación lingüística de los estudiantes que podrán trascender en campos empresariales, culturales y académicos con más oportunidad de éxito. Con la retroalimentación

19 Qiu Gui Su, «Learning Mandarin Chinese: A Step-by-Step Guide», About Education (2016), 22 mayo 2016, <http://mandarin.about.com/od/educationlearning/tp/learn_by_step.htm>. 
aportada por los profesores, se pretende proponer mejoras que contribuyan al plan de estudios del Bachillerato en Mandarín. La propuesta planteada busca favorecer aproximaciones innovadoras sobre la enseñanza de idiomas, métodos pedagógicos eficaces utilizados por otros profesores de idiomas, así como nuevas pedagogías para una educación de calidad. Se deben considerar algunos otros medios eficaces para mejorar la competencia lingüística, de tal manera que satisfagan las necesidades de aprendizaje de los alumnos que evolucionan constantemente.

El estudio contribuye a la propuesta de un bachillerato en mandarín, con lineamientos curriculares que podrían beneficiar a los estudiantes, preparar a los futuros profesores de esta carrera y favorecer las iniciativas para esta carrera futurista, parte de la realidad costarricense y latinoamericana. 
Anexo 1. Cuestionario

\begin{tabular}{|c|c|c|}
\hline$\frac{\text { UNA }}{\text { UNIVRSIDAD }}$ & $\begin{array}{l}\text { UNIVERSIDAD NACIONAL } \\
\text { FACULTAD DE FILOSOFÍA Y } \\
\text { LETRAS }\end{array}$ & \\
\hline \multicolumn{3}{|c|}{$\begin{array}{c}\text { Escuela de Literatura y Ciencias del Lenguaje } \\
\text { Cursos de Mandarín }\end{array}$} \\
\hline \multicolumn{3}{|c|}{$\begin{array}{c}\text { Cuestionario sobre las experiencias de los estudiantes de } \\
\text { mandarín en Costa Rica }\end{array}$} \\
\hline $\begin{array}{l}\text { Instrucciones ge } \\
\text { sobre las experien } \\
\text { la Universidad } \mathrm{Na} \\
\text { la opción que con } \\
\text { aprendizaje del m }\end{array}$ & $\begin{array}{l}\text { les: Este cuestionario persigue recole } \\
\text { de los estudiantes aprendiendo el idi } \\
\text { al. Para contestar este cuestionario } \mathrm{m} \\
\text { e más cercana a sus percepciones en } \\
\text { rín. }\end{array}$ & $\begin{array}{l}\text { información } \\
\text { la mandarín en } \\
\text { que y responda } \\
\text { proceso de }\end{array}$ \\
\hline
\end{tabular}

I. Lea los siguientes enunciados y marque el valor que crea que le corresponde a cada uno según su experiencia en el aprendizaje del mandarín en la UNA.

\begin{tabular}{|c|c|c|c|c|c|c|}
\hline & ENUNCIADOS &  & 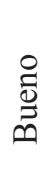 & 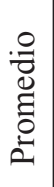 & 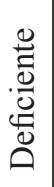 &  \\
\hline 1 & $\begin{array}{l}\text { ¿Cómo califica la enseñanza del idioma mandarín en } \\
\text { la UNA? }\end{array}$ & & & & & \\
\hline \multicolumn{7}{|c|}{ Justifique } \\
\hline 2 & $\begin{array}{l}\text { ¿Qué tan bien están planeados los cursos de mandarín } \\
\text { de la UNA? }\end{array}$ & & & & & \\
\hline \multicolumn{7}{|c|}{ Justifique } \\
\hline 3 & $\begin{array}{l}\text { ¿Cuánta utilidad tienen las dinámicas desarrolladas en } \\
\text { la clase de mandarín para su aprendizaje del idioma? }\end{array}$ & & & & & \\
\hline
\end{tabular}




\begin{tabular}{|l|l|l|l|l|l|}
\hline 4 & $\begin{array}{l}\text { ¿Es el aprendizaje del idioma mandarín beneficioso } \\
\text { para su formación profesional? }\end{array}$ & & & & \\
\hline Justifique \\
\hline 5 & $\begin{array}{l}\text { ¿Qué importancia tiene el aprendizaje del mandarín } \\
\text { como segundo idioma para su formación integral y } \\
\text { profesional? }\end{array}$ & & & & \\
\hline Justifique
\end{tabular}

II. Conteste las preguntas formuladas a continuación. Anote ejemplos y/o suministre explicaciones detalladas para completar sus respuestas.

1. ¿Qué estrategias de aprendizaje utilizó para superar la distancia cultural y lingüística del idioma mandarín?

2. ¿Cuál es su motivación para aprender este idioma?

3. ¿Qué tipo de actividades de clase son las que más le han ayudado en el aprendizaje del mandarían y por qué?

4. ¿Qué tipo de actividades de la clase son las que menos le han ayudado en el aprendizaje de mandarín y por qué?

5. ¿Cuáles cree usted son las ventajas de aprender el idioma mandarín? Puede marcar más de una opción.

$\square$ Trabajo laboral

$\square$ Formación profesional

$\square$ Completar un requisito de mi programa de estudios universitarios

$\square$ Adquirir conocimientos culturales

$\square$ Otras razones. Especifique:

6. Otros comentarios. Anote cualquier otra información que usted piense pueda ser útil en esta investigación. 